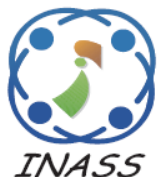

\title{
Breast Cancer Detection in Mammogram Images by MapReduce Based Deep Convolutional Neural Networks
}

\author{
Nagalakshmi Thirunavukkarasu $^{1 *}$ Govindarajan Muthukumarasamy ${ }^{1} \quad$ Ramalingam Murugesan $^{2}$ \\ ${ }^{I}$ Department of Computer Science and Engineering, Annamalai University, Tamilnadu, India \\ ${ }^{2}$ Department of Information Technology, Mailam Engineering College, Villupuram,Tamilnadu, India \\ * Corresponding author's Email: lakshusenthilphd@gmail.com
}

\begin{abstract}
Imaging techniques using mammographic pictures are the most efficient and straightforward way to diagnose Breast Cancer (BC). The accurate discovery can significantly lower the mortality rate caused by BC. Machine Learning (ML) techniques were utilized for the prediction of BC in images. The ML approaches offered a unique way of making decisions, and they were able to aid the medical expert in providing a second perspective on more precise nodule detection. However, the over-fitting problem in ML degraded the performance of BC detection. To solve this issue, Deep Learning (DL) algorithms were utilized to detect BC early using mammographic images, which is a huge help in detecting BC at an early stage. The Deep Convolutional Neural Networks (DCNN) are being used in DL to help with an accurate BC diagnosis and better BC image outcome detection. However, the time complexity of DCNN is high for a large-scale dataset. In this paper, The DCNN is parallelized in the Mappers of MapReduce (MR) programming model in which the network weights can be iteratively adjusted via determining their fractional gradients following all collections of learning images are propagated throughout the system. Accordingly, the Mapper will parallelize the training phase by distributing the normal, benign, and malignant images that have been preprocessed using the K-Means (KM) algorithm. Each image can be provided to various DCNNs and each DCNN is trained independently in parallel. The output weight of the training stage of DCNNs is aggregated in Reducer and then the weights are then updated for the next iteration. After the completion of the training process, the updated weight is utilized in the all distributed DCNN for classifying the test images of BC. The whole process is termed MapReduce-KM-DCNN (MR-KM-DCNN). Finally, the experimental outcome demonstrates that the proposed MR-KM-DCNN method achieves 88.35 percent accuracy, which is $13.48 \%, 8.02 \%$, and $4.85 \%$ higher than the existing SSL-DCNN, AN-DCNN, and KM-DCNN methods. In addition, the running time is reduced by $60 \%$ on average compared to previous methods. As a result, it has been verified that the MR-KM-DCNN attained the highest BC detection rate while requiring the smallest amount of computational time.
\end{abstract}

Keywords: Breast cancer detection, Mammogram images, Deep convolutional neural networks, MapReduce programming model.

\section{Introduction}

Breast cancer is a condition that causes malignant (cancer) cells to develop in the breast tissues. Immunotherapy can be highly successful, particularly when an illness is initially diagnosed [1]. When a patient is diagnosed with $\mathrm{BC}$, a stage is allocated to them so that doctors can provide the best therapy and prognosis at an early stage. BC stages are classified as in situ (non-invasive) or invasive (invasive) and are assigned a number from
0 to IV. BC is typically diagnosed through a biopsy of nodules discovered utilizing medical equipment such as mammography or palpitation. This practice has greatly increased the detection of early-stage tumors, sometimes these methods eluded physicians until cancer had progressed to more lethal stages [2].

The imaging technologies used to obtain BC images include machine-assisted diagnostics, computerized radiology, MRIs and ultrasonography. These include the quickest and precise way of collecting pictures of BC [3]. An advancement of imaging techniques is being used to increase the 
detection rate of $\mathrm{BC}$ as well as reducing unnecessary biopsy.

Using a ML classification for the image of $\mathrm{BC}$, physicians can take a second view and satisfy them and boost the patient's trust. Few professionals can provide a precise evaluation of the illness. The machine-based predictive method can help patients to receive an instant response from diseases and therefore enhanced healthcare practices.

The use of AI techniques in conjunction with image processing [4] has resulted in considerable improvements in the diagnostics and classification of BCs from images. Noise smoothing, bad pixel replacement and Vignetting are the methods used for pre-processing images. Edge detection, regiondependent and thresholding methods are used for segmenting images. Color features, edge features, shape and texture are extracted from segmented images. The ML methods like k-NN, ANN and SVM are used for diagnosing BCs. The appropriate pre-processing, segmentation, feature extraction and ML methods for classification are chosen based on the quality and type of acquired input images.

However, the ML method has many limitations, including a slow convergence speed and a tendency to become stuck at the local minimum. To address this problem, a multi layer perceptron (MLP) neural network training technique based on the whale optimization algorithm is suggested [5]. In the MLP neural network, the optimization technique is used to reduce the error rate of the training stage, which includes false negatives and false positives. However, the performances of optimized ML also are affected due to the problems due to problems caused by imprecise image processing outputs such as boundary segmentation and feature robustness. The relevant feature selection and parameter optimization are other common difficulties.

The DL technique provides better detection results for BC images than ML approaches. DCNN is proposed to classify screen-film and digitalized mammogram input images by multitask transfer learning. When compared to the single-task transfer learning DCNN, the multi-task exchange training DCNN had a much longer execution time [6].

In this paper, MR-KM-DCNN technique is proposed to achieve an efficient detection of BC. The DCNN is parallelized in the MR programming model. DCNN is allowed to run independently in Mapper. The training images are pre-processed by $\mathrm{KM}$ and then the training images are distributed to DCNNs. The generated weights in all DCNNs are aggregated in Reducer, and then the aggregated weight is again distributed to all mappers to iteratively adjust weight values through computing partial gradients. The weighted model generated in the training phase is utilized in the testing phase. In the testing phase, test images are pre-processed by $\mathrm{KM}$ and distributed to all mapper. The predicted result of test images from all mappers are aggregated and obtained predicted results from reducer. Hence, the proposed method improves the computational time of $\mathrm{BC}$ detection for large-scale image sets while maintaining the high classification accuracy rate.

The rest of the manuscript is organized as the following. $\mathrm{CNN}$ used for $\mathrm{BC}$ detection from breast images is explained in section 2. The suggested MRbased CNN is explained in section 3, followed by a set of tests undertaken and reported in section 4 and lastly the study is completed in section 5 .

\section{Literature survey}

A KM-DCNN [7] was proposed with MSVM for $\mathrm{BC}$ detection from images. KM was initially used to segment for eliminating noises and refining boundary of objects. The preprocessed images were then send to DCNN.DCNN extracted features from images and MSVM processed classifcation. However, the time taken for processing images is high.

A DL system for automatic image feature extraction utilizing shear-wave elastography was developed [8] for separating benevolent and malevolent BCs. A two-layer DL structure was created with the point-wise gated and constrained Boltzmann machines to extract the features. Hidden units in the structure were process-appropriate and inappropriate, with the process-appropriate modules linked to the constrained Boltzmann machine. On the other hand, the computational complexity was high.

An adapted AlexNet [9] has been created for the MIAS database to classify BC as benign or malignant. Mammographic have an outlier, variable resolutions, and various intensities, making it difficult to locate tumor tissue. The Gaussian filtration was applied in pre-processing to reduce outliers. Finally, image enrichment was used for augmenting the images to learn CNN efficiently. However, involving different operations like reflection and rotation in data augmentation is additional overhead.

A semi-supervised DCNN was developed [10] to improve the accuracy of diagnosing $\mathrm{BC}$ for a large amount of unlabeled data. Diving and cotraining processes were incorporated in the DL algorithms for analyzing the cancer images. This method required a small amount of labeled data with 
an exploit abundant resource of unlabeled data. On other hand, when the amount of labeled data increased, the performance became slow.

The deep CNN model DenseNet-201 was proposed [11] for diagnosing $\mathrm{BC}$ using medical histology. The features from the histopathology pictures were mined using the multi-layered feature extraction technique. The features were extracted using a dense CNN, which assisted the structure in training using attributes for enhancing image categorization. However, the problem of overfitting could be caused by a small training set.

A machine-assisted diagnostics framework depending on ROI-based CNN was proposed [12] to handle recognition and categorization of $\mathrm{BCs}$. The proposed CAD system employs convolutional units subsequent to the fully connected neural networks (FC-NNs) for recognizing the right position of the cancer tissues and categorizing benevolent and malevolent BCs.

A supervised approach was presented [13] to detect the mitotic signal from breast histopathology whole slide images (WSI) images. The model was built using DL architecture to train and classify from a huge number of non-annotated histological breast imaging data. The DLN characteristics had been combined with the handcrafted features. The features were then fed straight into the fully connected layer for high-level image representation of mitosis and non-mitosis images. However, in each of the mitosis phases, the shape of mitotic figures was varied, resulting in a higher falsepositive rate.

A multi-objective feature selection technique was developed [14] for the BC detection by. Initially, data augmentation was applied to extract morphological features like HoG and LTP of the images. Then, the features were extracted from images by using CNN.Then; relevant features were selected by multi-objective feature selection method. The selected features were transferred to the LSTM network for BC classification. However, this method was resulted with low convergence rate.

The patch-based CNN model was presented [15] to classify and grading malignant. The patch-based CNN model was trained with the unsurpassed parameters extracted from the pre-processed biopsy images. The standard convolution filters, activation functions, pooling operations were utilized from different transfer learning models to construct this model. This developed would assist the pathologists to solve inter-observer variability problem which arise while grading tumours. However, this method would require large data for training.

A BC prediction model was constructed [16] by
$\mathrm{KM}$ and SVM.The modified $\mathrm{KM}$ was used to construct a high quality training datasets. Then, the SVM was trained and used to classify the malignant cases of unknown images. However, classification accuracy is required to improve.

The hybrid feature extraction technique was developed [17] to overcome the misclassification issues of BCs detection in MRI scanned images. Contrast based histotogram method was used to remove the noises. Multilevel Otsu's thresholding was used to remove unnecessary breast tissues. Haralick oriented pattern and haralick texture feature were extracted. DL methods were used to classify malignant or benign. However, the hybrid features were used in this method results with high system complexity due to the optimization problems.

The modified active contour technique was developed [18] for the detection of BC. Snakes and level sets were applied to active contour for creating a clear contrast between the tumour and the normal breast region. Based on the segmented region's characteristics, Chan-vese method (C-VM) was used to accurately determine the location and shape of the $\mathrm{BC}$ boundaries. This method required large number of iterations, because its bias was limited in one direction only, either inside or outside the region of interest.

However, all of the above methods are not implemented parallel. The processing of every individual image takes time in minutes. The main scientific contribution of this paper is reduce the time by parallelly processing images. Map reduce methodology is used to implement the $\mathrm{KM}$ and DCNN to run parallelly in many nodes.

\section{Proposed methodology}

The proposed MR-KM-DCNN technique for predicting the $\mathrm{BC}$ images is described below in detail. In this proposed method, the DCNN has been used to benefit the parallelized computation obtained using MR programming structure [19]. The DCNN model is performed in each mappers to weight initialization and updating for training dataset. Thus, following preprocessing with KM, parallelization in the learning step is achieved via dividing the mammography pictures into many groups [20]. The KM segmentation smoothen the images by clustering the pixels closest to them by $\mathrm{K}$ points in the space. After that, each distributed image is given to the DCNNs, and every DCNN is learned separately. The outcomes are combined to provide the main findings that are applied in the subsequent epoch to adjust the weights. The outcomes are then merged to get the completed 
outcomes used to adjust parameters for the next epoch.

The mappers in the MR framework use the training images as input as $(x ; y)$, as well as a set of random initialization weights is given by $\left[w_{1}, w_{2}, \ldots \ldots \ldots \ldots, w_{n}\right]$. The weights set contains $\mathrm{n}$ weights, which correspond to the amount of hidden layers. As a result, $w_{(i)}$ associates with the hidden layer's weight $i$. As a result, the learning image is referred to as a tuple collection in the condition of $(x, y)$, where $x$ signifies the point of training and $y$ signifies the classified label for the prediction of $\mathrm{BC}$ datasets (normal, malignant, and benign).The constructed network is initialized by each mapper with the provided weight set and the network is

Table 1

\begin{tabular}{|c|c|c|c|}
\hline $\begin{array}{l}\text { variable } \\
\text { /functions }\end{array}$ & description & Location & $\begin{array}{c}\text { Algorith } \\
\text { m }\end{array}$ \\
\hline $\mathrm{x}$ & input images & $\begin{array}{c}\text { Mapper } \\
\text { and reducer }\end{array}$ & $\begin{array}{l}\mathrm{KM} \text { and } \\
\mathrm{DCNN}\end{array}$ \\
\hline $\mathrm{y}$ & label of image & $\begin{array}{c}\text { Mapper } \\
\text { and reducer }\end{array}$ & $\begin{array}{l}\mathrm{KM} \text { and } \\
\mathrm{DCNN}\end{array}$ \\
\hline $\mathrm{w}_{1}$ to $\mathrm{w}_{\mathrm{n}}$ & $\begin{array}{c}\text { weight values } \\
\text { of layers in } \\
\text { CNN }\end{array}$ & $\begin{array}{c}\text { weight } \\
\text { calculated } \\
\text { in Mapper } \\
\text { and } \\
\text { aggregated } \\
\text { in reducer }\end{array}$ & DCNN \\
\hline$\Delta w_{1}$ to $\Delta w_{n}$ & $\begin{array}{c}\text { changing of } \\
\text { weight }\end{array}$ & $\begin{array}{l}\text { Mapper } \\
\text { and } \\
\text { updated in } \\
\text { reducer }\end{array}$ & DCNN \\
\hline$M A P()$ & call by Mapper & Mapper & DCNN \\
\hline Reduce () & call by reducer & reducer & DCNN \\
\hline Driver () & $\begin{array}{l}\text { call from } \\
\text { master node } \\
\text { for whole } \\
\text { process }\end{array}$ & Mapper & DCNN \\
\hline $\begin{array}{l}\text { updated } \\
\text { weights }\end{array}$ & $\begin{array}{c}\text { newly } \\
\text { calculated } \\
\text { weights are } \\
\text { updated to } \\
\text { reducer }\end{array}$ & Mapper & DCNN \\
\hline $\begin{array}{c}\text { sum } \\
\text { Weights }\end{array}$ & $\begin{array}{l}\text { weight from } \\
\text { all hidden } \\
\text { layer of } \\
\text { DCNN is } \\
\text { summed in } \\
\text { reducer }\end{array}$ & reducer & DCNN \\
\hline Final Weigh & $\begin{array}{c}\text { used in } \\
\text { Mappers again } \\
\text { for prediction } \\
\text { reducer }\end{array}$ & Mapper & DCNN \\
\hline
\end{tabular}

utilized for training the entry images. The outcome resulted in a group of new range updated weights
$\left[\Delta w_{1}, \Delta w_{2}, \ldots \ldots \ldots, \Delta w_{n}\right]$ then it is processed to the reducer. The mappers obtain a series of modified weights in each iteration, in which they are subsequently distributed around the network in order to reach the maximum number of iterations possible. Parameters and function are described in Table1.

The input images are segmented by KM. Then the images are sent to various DCNN running in different nodes. The Map() function generate the weight values of each DCNN during training, the calculated weight in each mapper is updated, then reducer aggregate and summed the weight. Driver function update the weight after iteration and newly trained images. Finally updated weight is used as a model for predicting unknown image classes. This is explained in three algorithms.

The pseudo code for the Mapper operation is given in the Algorithm 1.

\section{Algorithm 1: Mapper in MR-based KMDCNN}

Input: The Mapper is a collection of tuples $(x ; y), x$ is a training image and $y$ is the classified label for the prediction (Normal, malignant and benign) of $\mathrm{BC}$ datasets. For each hidden layer, the weights are a collection of arbitrarily initialized weights $i$.

Function MAP ()

Examine weights from Hadoop;

Initialize CNN with arbitrary weights;

for $i \leftarrow 0$ to num layer -1 do

updated Weights [i]

updated Weights [i] + Weights

\section{Output: updated weights}

The reducer aggregates the weights generated by each mapper using the transitional outcome from the mappers as input. The reducer will readily collect weights based on their index because the weight's arrangement in the performance of mappers is ordered by the hidden unit to which they belong. To build an average of weights, the accumulation determines the total weights, which is divided using the learning examples in the batch. The absolute outcome is utilized for modifying the network weights before being passed back to the Mapper for the consecutive epoch. Algorithm 2 describes the reducer operation.

\section{Algorithm 2: Reducer in MR-based KMDCNN}

Input: The reducer is transitional outcome of Mappers wherein the modified weights for each hidden layer (numLayers) are given.

sumWeights is the collection that accumulates 
the total weights for all hidden units.

Function Reduce ()

sumWeights $\leftarrow$ Initialize list of zeros;

for $i \leftarrow 0$ to numLayer -1 do

$$
\text { sumWeights }[i] \leftarrow \text { sumWeights }[i]+
$$

Weights

(2)

\section{Output: sumWeights}

The MR structure makes use of the driver to serve as a task scheduler. The driver generates a directed acyclic graph (DAG) or execution plan for the programme, which is then broken into smaller jobs to be carried out. The driver communicates with hadoop to decide which map and reduce groups to utilise and to configure the work module. The configurations are provided by the user, and they include the road to the training data, the path of performance, training parameters, and network setups.

The training set specifications include the quantity of learning images, the quantity of validation images, the maximum epochs and the batch size. Network parameters include the size of the receptive field, stride, number and shape of layers, training fraction and optimization strategy. Algorithm 3 depicts the pseudocode for the driver operation.

Algorithm 3: Driver in MR-based KMDCNN

Function Driver()

for layer $\leftarrow 0$ to numLayers -1 do

Weights[layer] $\leftarrow$

Randomly InitializedWeights

numiteration $\leftarrow 1$

while numIteration $<=$ maxIterations do

Initialize MR Job;

Store weights in HDFS;

Pass model parameters to MR Job;

Run MR jobs:

Input for Mappers: Weights for each hidden layer;

Mappers compute updated weights for every hidden layer;

Output of Mappers: Newly trained weights;

Aggregate Weights of each hidden layer:

Input to Reducer: Newly trained weights from Mappers;

Reducers aggregate weights for all hidden units:

for layer $\leftarrow 0$ to numLayers -1 do

$$
\text { Weights[layer] } \leftarrow
$$

Weights[layer] + updatedW eights[layer]
Outcome of Reducer: collective amount of weights for all hidden units;

Update weights of each hidden layer;

numIteration + +;

Output: Final Weights

This Algorithm 3 describes the driver of DL in MR program. Once the training process completed, the finally updated weight is utilized for proposed classifying test images by the same procedure. Instead of weight generation, the generated weight is utilized in the network to predict the outcome of given test image. Finally, test image results are evaluated for classification accuracy and ROC results. Fig. 1 portrays an overall work of the proposed $\mathrm{BC}$ prediction.

\section{Details of DCNN and MR architecture}

The DCNN architecture employed in the tests is a VGGNet [21] modification. The input is $255 \times 255$ RGB pictures in which 350 are applied for learning and validation. The maximum amount of learning epochs is assigned to 100 . There is no data augmentation, and the only pre-processing step is KM clustering.

The network is made up of 12 weighted layers, which include 10 convolution units and $2 \mathrm{FC}$ units. The initial layer has 64 channels, which doubles with each max-pooling layer until the final convolution layer has 512 channels. Throughout the network, minute $6 \times 6$ receptive fields and stride 1 are used. The ReLU non-linearity follows each convolution layer. Every weighted layer employs the initialization [22]. Following the stack of

Table 2. DCNN architecture layers

\begin{tabular}{|c|c|c|c|}
\hline $\begin{array}{c}\text { CNN } \\
\text { Architecture }\end{array}$ & $\begin{array}{c}\text { Size of the } \\
\text { corresponding } \\
\text { field }\end{array}$ & Channel & $\begin{array}{c}\text { No. of } \\
\text { units }\end{array}$ \\
\hline $\begin{array}{c}\text { Convolution } \\
\text { unit }\end{array}$ & 3 & 64 & 3 \\
\hline MaxPool 1 & 3 & 128 & 3 \\
\hline MaxPool 2 & 3 & 256 & 3 \\
\hline MaxPool 3 & 3 & 512 & 3 \\
\hline FC unit 1 & - & 400 & 1 \\
\hline FC unit 2 & - & 3 & 1 \\
\hline
\end{tabular}




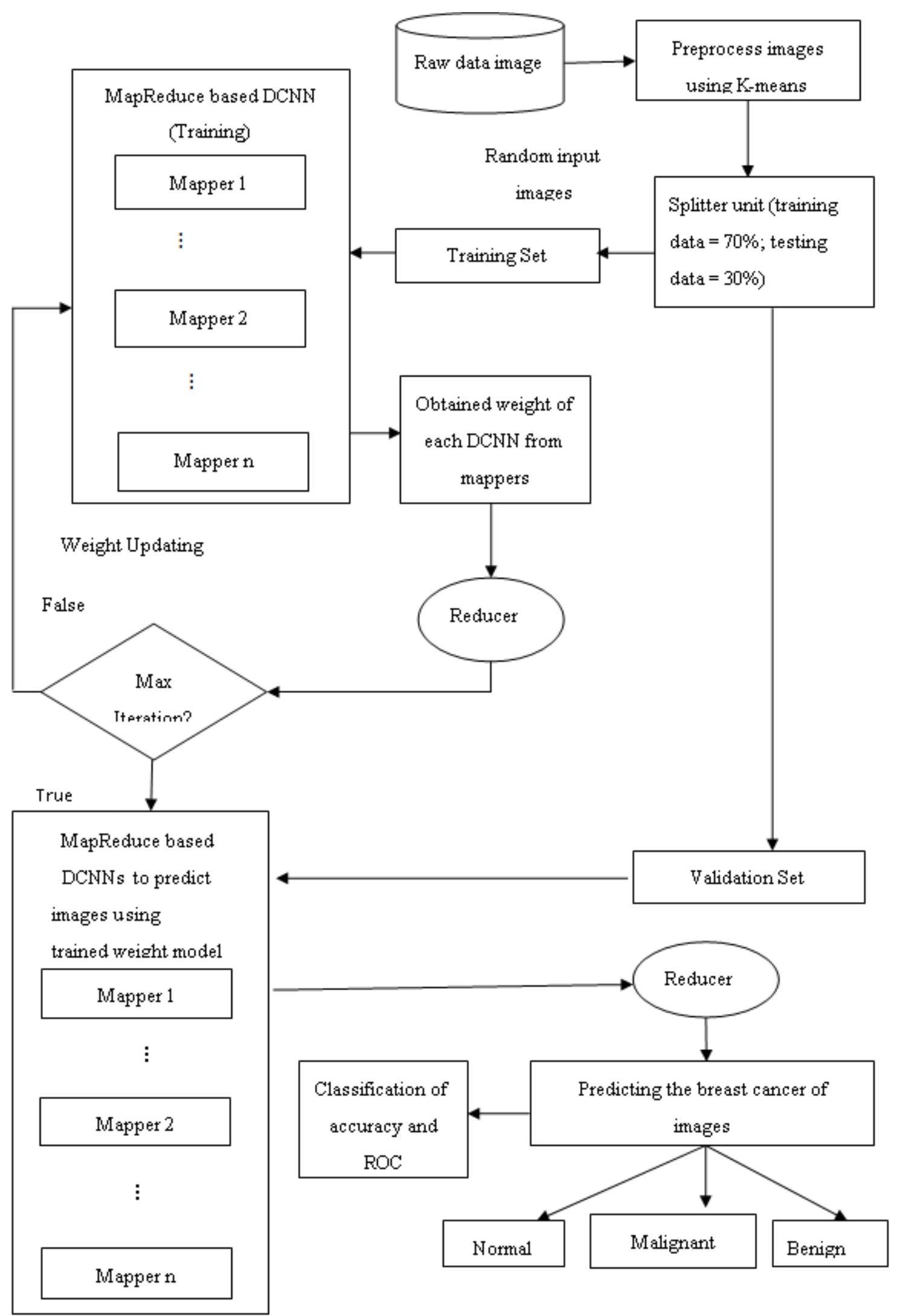

Figure. 1 Overall work of the proposed BC prediction 
Table 3. Preprocessed output of normal BC images

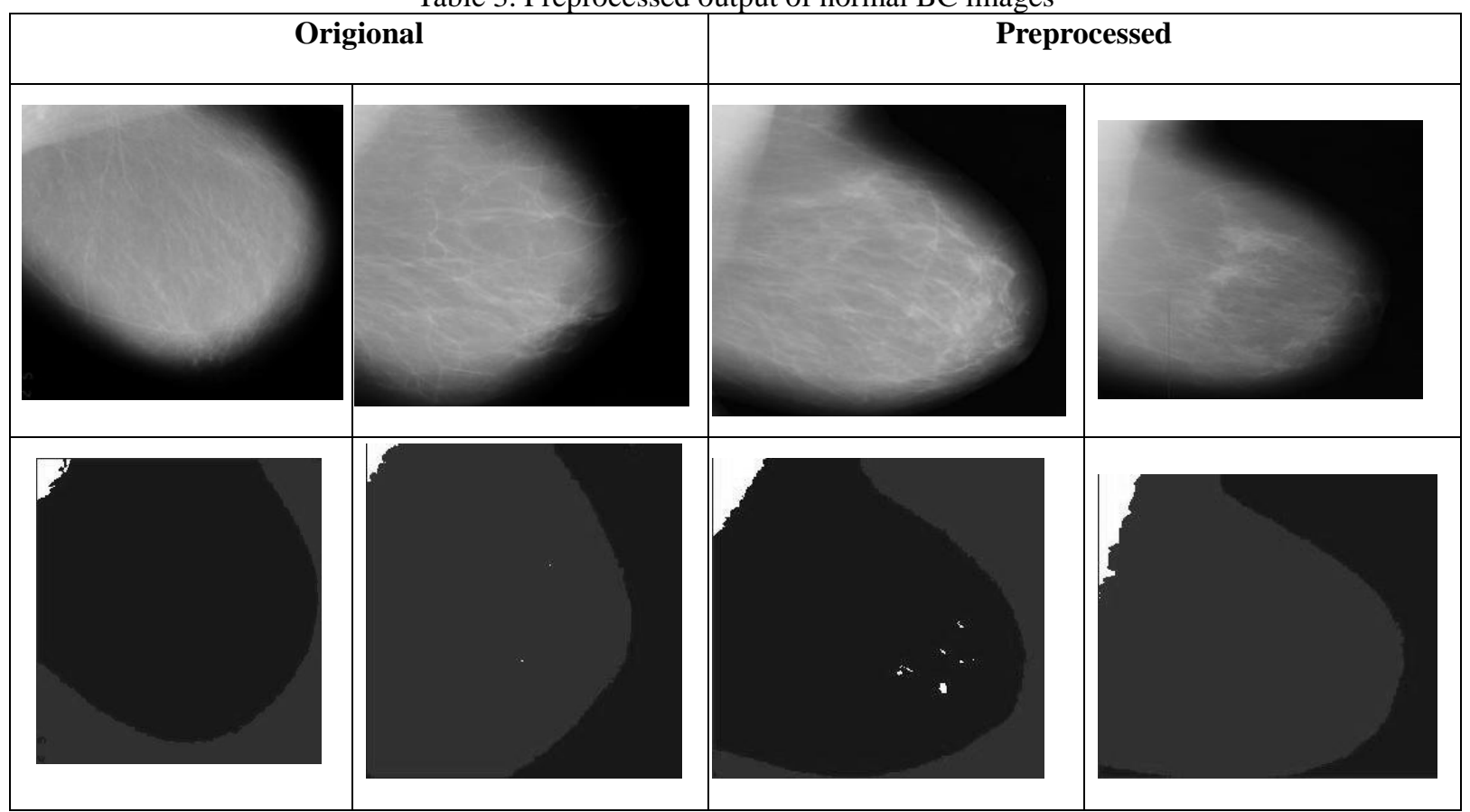

The mappers are used for $\mathrm{BC}$ image classification from 2 to 10 nodes. A reducer is implemented in a single node. The configuration of name node (master node) and data node (slave nodes) for convolution layers, 2 FC units are added. The first layer comprises 400 channels, while the second has three channels for each of the MIAS dataset's three classes. The soft-max layer is the final layer, which is used to determine category output and class probability. The DCNN architecture is shown in Table 2. implementing MR configuration is Intel Pentium core i5, $2.5 \mathrm{GHz}$ CPU, with 8GB RAM, Windows 10 operating system. The entire work is implemented using MATLAB 2017b.

The performance of the proposed work is measured for classification accuracy and computation time. The classification metrics are accuracy, precision, recall and F-measure. The time is taken from the computer before and after processing classification.

Accuracy is the fraction of accurately classified images in each category of $\mathrm{BC}$ and normal images over the sum amount of breast images considered.

$$
\text { Accuracy }=\frac{T P+T N}{T P+T N+F P+F N}
$$

TP (True Positive):

No. of exactly classified breast cancer image samples

FP (False Positive):

No. of exactly classified normal breast image samples

FN (False Negative):

No. of inexactly classified normal breast image samples

TN (True Negative):

No. of inexactly classified breast cancer image samples

Precision is the ratio of exactly classified categories of $\mathrm{BC}$ images, normal images. with totally classified breast images.

$$
\text { Precision }=\frac{T P}{T P+F P}
$$

A recall is the ratio of exactly classified categories of $\mathrm{BC}$ images with totally classified images as $\mathrm{BC}$.

$$
\text { Recall }=\frac{T P}{T P+F N}
$$

F-measure is the harmonic average of precision and recall.

$$
F-\text { measure }=2 \times \frac{(\text { Precision } \cdot \text { Recall })}{(\text { Precision }+ \text { Recall })}
$$

The association between the false positive rate (FPR) and the true positive rate (TPR) is known as the ROC curve. The values of ROC measured from 0 to 1 . 


\section{Result and discussion}

In this section, the proposed MR-KM-DCNN is compared with existing semi-supervised learning DCNN (SSL-DCNN) [9], AlexNet DCNN (ANDCNN) [10] and KM-DCNN [7] for improving the best classification accuracy of $\mathrm{BC}$ with less computational time. For the experiment analyses, the mini mammographic database is collected from the mammographic image analysis society (MIAS) database [23]. This dataset has 350 images. The supporting file in this database contains information like reference number to breast images, the character and the type of the background tissue, class of anomaly presented, severity of the abnormality, the $\mathrm{x}, \mathrm{y}$ image-coordinates of the abnormality's centre, and the approximate radius (in pixels) of a circle enclosing the abnormality. These details are very helpful to train the classifier effectively. The performance in Map reduce-KMDCNN for classifying three different BC types like normal, benign and malignant images are shown in Table 3, 4 and 5.

Table 4. Preprocessed results of benign $\mathrm{BC}$ images

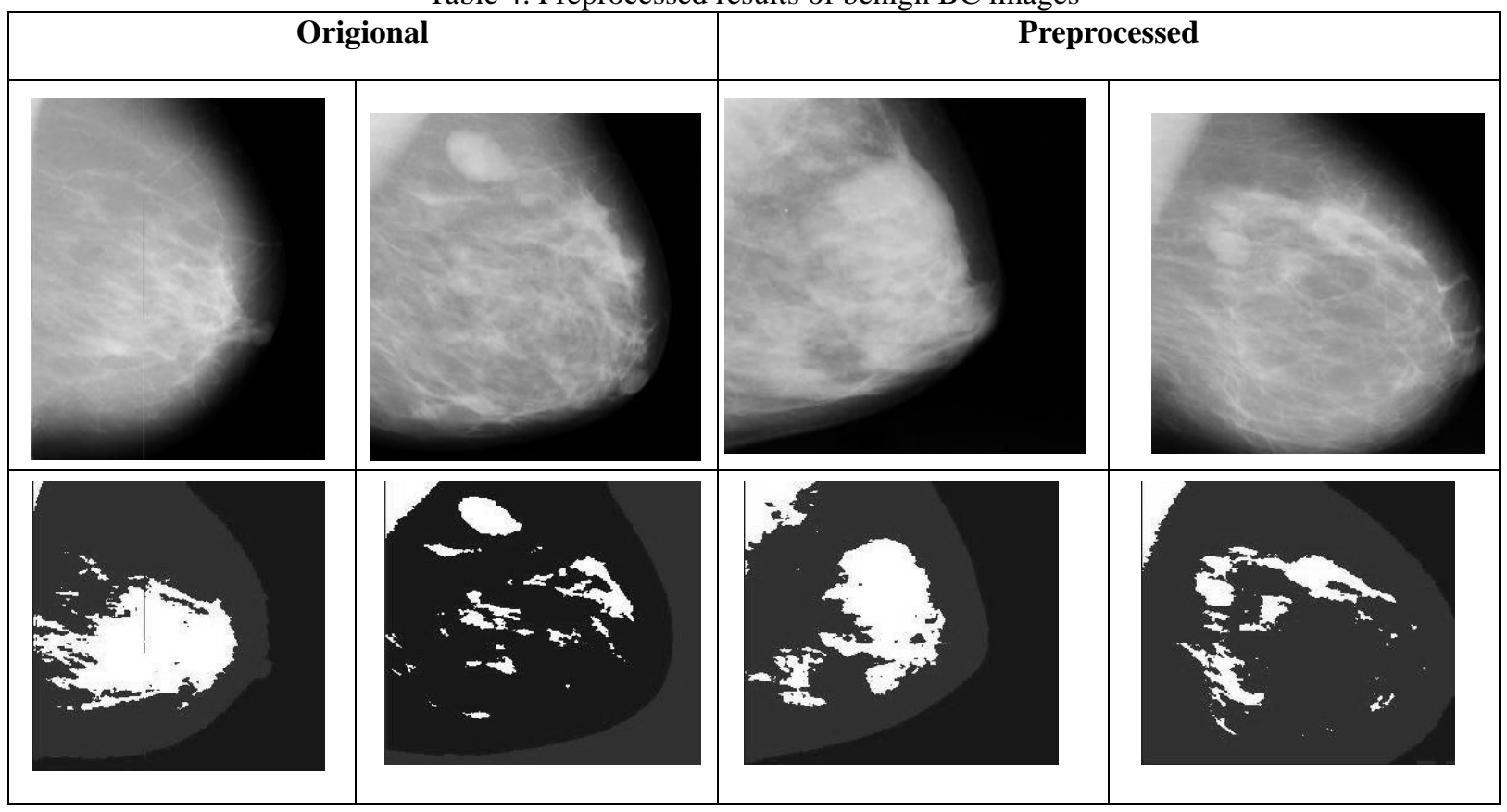

Table 5. Preprocessed result of malignant BC images

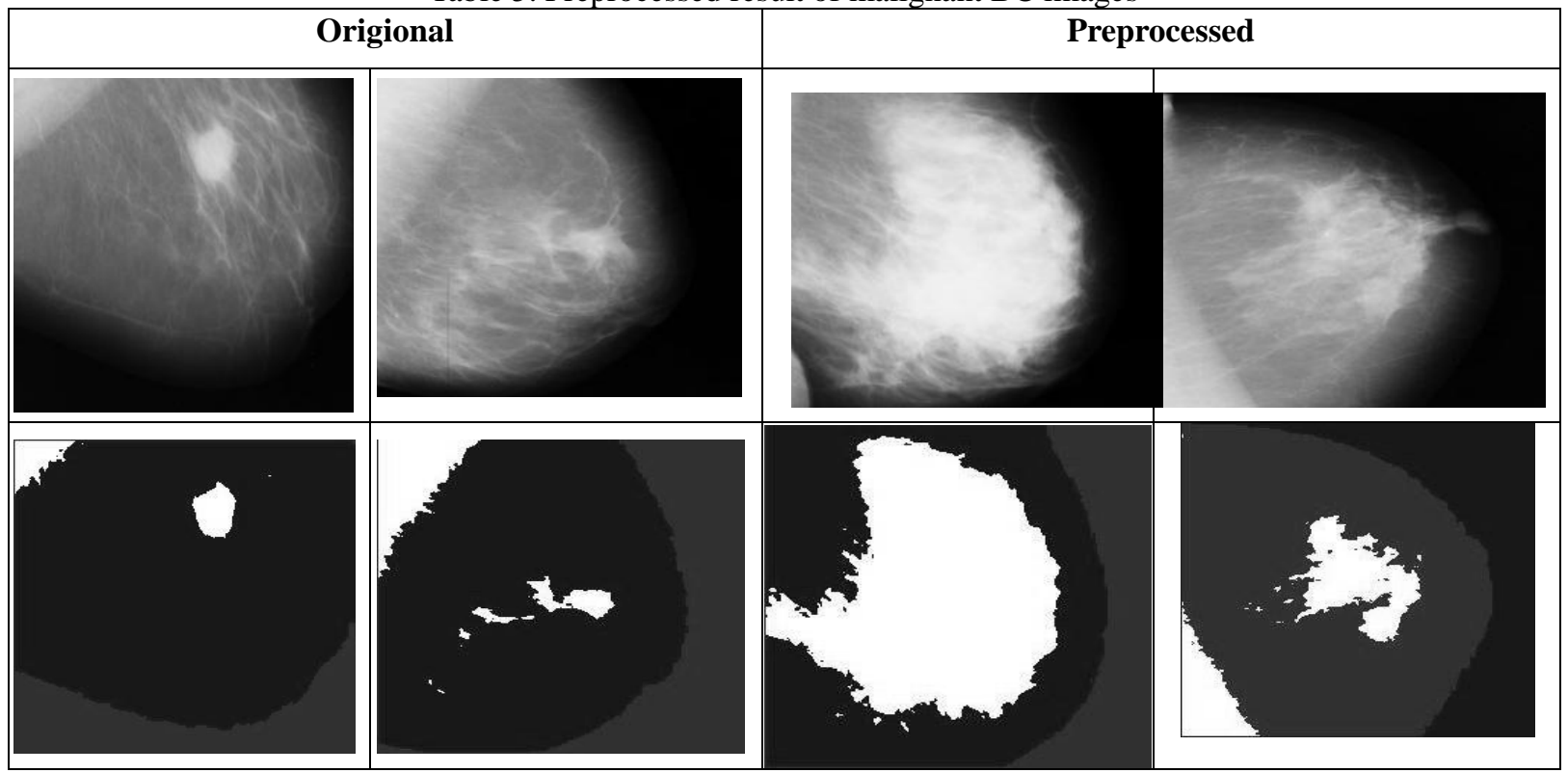




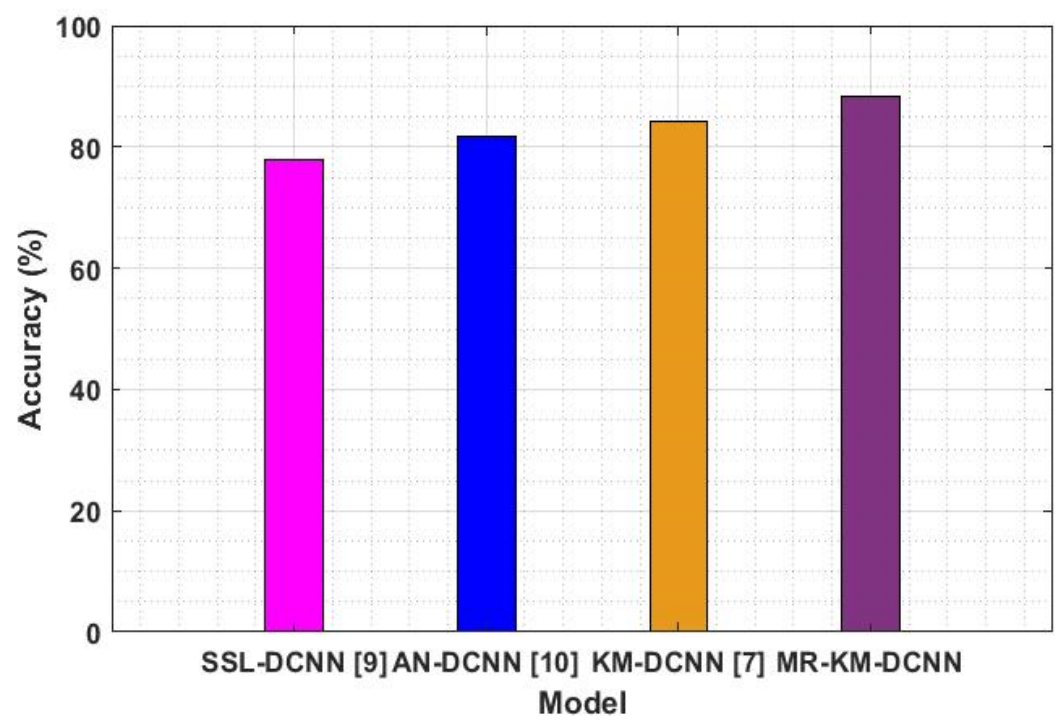

Figure. 2 Comparison of accuracy

Table 6. Comparison of accuracy values

\begin{tabular}{|c|c|}
\hline Methods & Accuracy Values \\
\hline SSL-DCNN [9] & 77.85 \\
\hline AN-DCNN [10] & 81.79 \\
\hline KM-DCNN [7] & 84.26 \\
\hline MR-KM-DCNN & 88.35 \\
\hline
\end{tabular}

Table 7. Comparison of precision value

\begin{tabular}{|c|c|}
\hline Methods & Precision Values \\
\hline SSL-DCNN [9] & 75.34 \\
\hline AN-DCNN [5] & 78.56 \\
\hline KM-DCNN [7] & 80.05 \\
\hline MR-KM-DCNN & 85.27 \\
\hline
\end{tabular}

In this experiment, many parameters such as the number of training samples, validation samples, the maximum number of iterations, and configurations are provided to train the $\mathrm{BC}$ images. In the DCNN structure, the image performs $50 \%$ training and $50 \%$ testing. The proposed method is evaluated with different performances to improve the classification accuracy of the BC images for efficient detection and diagnosis.

The accuracy values of the SSL-DCNN, ANDCNN, KM-DCNN and MR-KM-DCNN algorithms are compared in Fig. 2 and Table 6 from this analysis, it is calculated that the proposed MRKM-DCNN is $13.48 \%, 8.02 \%$ and $4.85 \%$ is greater than the existing SSL-DCNN, AN-DCNN and KM-DCNN. It is due to the MR programming model which provides the added benefit of improved generalized training and testing images

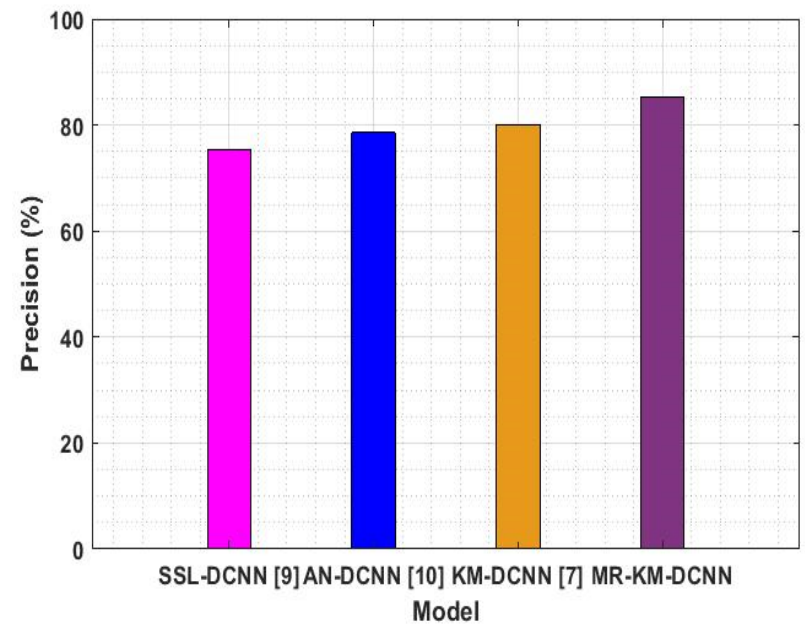

Figure. 3 Comparison of precision values

for higher validation accuracy. Hence, it has been demonstrated that the proposed strategy will outperform all other existing methods for predicting BC images in terms of accuracy.

Fig. 3 and Table 7 represent the values of SSLDCNN, AN-DCNN, KM-DCNN and MR-KMDCNN algorithms. From this analysis, it is calculated that the proposed MR-KM-DCNN is $13.18 \%, 8.5 \%$ and $6.5 \%$ is greater than the existing SSL-DCNN, AN-DCNN and KM-DCNN. It is due to the DCNN model which augments the training data in difficult cases and is highly focused on the misclassified BC images. So, it is proved that the proposed method can achieve better precision compared to all other existing methods.

Fig. 4 and Table 8 depict the comparison recall 
Table 8. Comparison of recall value

\begin{tabular}{|c|c|}
\hline Methods & Recall Values \\
\hline SSL-DCNN [9] & 77.84 \\
\hline AN-DCNN [10] & 78.41 \\
\hline KM-DCNN [7] & 81.03 \\
\hline MR-KM-DCNN & 86.55 \\
\hline
\end{tabular}

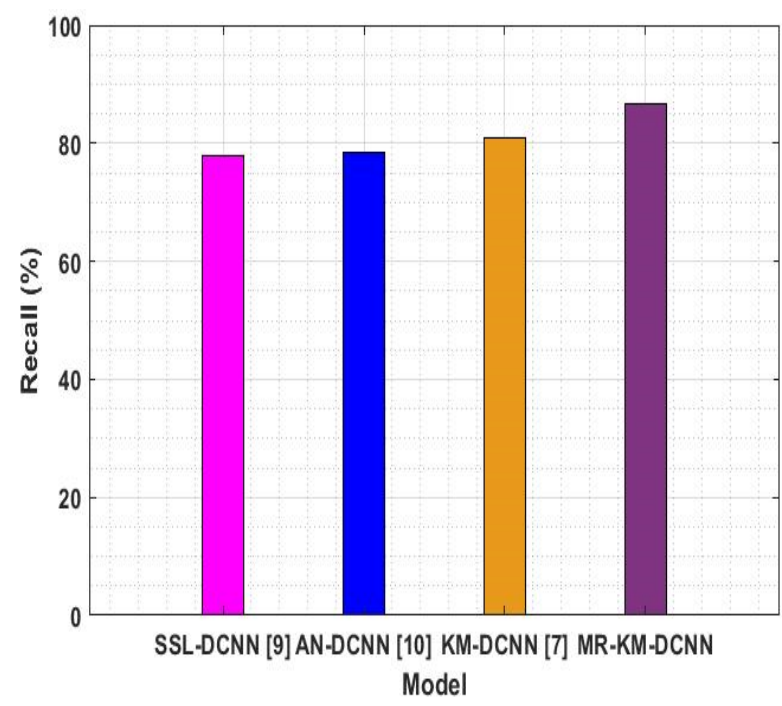

Figure. 4 Comparison of recall value

Table 9. Comparison of F-measure values

\begin{tabular}{|c|c|}
\hline Methods & F-measure values \\
\hline SSL-DCNN [9] & 75.82 \\
\hline AN- DCNN [5] & 78.41 \\
\hline KM-DCNN [7] & 81.03 \\
\hline MR-KM-DCNN & 86.55 \\
\hline
\end{tabular}

values among SSL-DCNN, AN-DCNN and KMDCNN and MR-KM-DCNN algorithms.

From this analysis, it is calculated that the proposed MR-KM-DCNN is $13.49 \%, 9.51 \%$ and $7.11 \%$ is greater than the existing SSL-DCNN, MAN-DCNN and KM-DCNN. The proposed MRKMDCNN provides the ratio of accurately classified $\mathrm{BC}$ images that emerged out to the total number of classified BC images. So, it is proved that the proposed method can achieve better recall compared to other existing methods for predicting $\mathrm{BC}$ images.

Fig. 5 and Table 9 display the comparison Fmeasure values among SSL-DCNN, AN-DCNN, KM-DCNN and MR-KM-DCNN methods. From this analysis, it is calculated that the proposed MR$\mathrm{KMDCNN}$ is $14.15 \%, 10.38 \%, 6.81 \%$ is greater than the existing SSL-DCNN, AN-DCNN and KMDCNN. It is due to the KM algorithm which eventually distributes the $\mathrm{BC}$ images to the training phase resulting in the exact images with higher Fmeasure. So, it is proved that the proposed method can achieve better F-measure compared to all other existing methods for predicting BC images.

Fig. 6 represents the comparison ROC values between SSL-DCNN, AN-DCNN, KM-DCNN and MR-KM-DCNN methods. From this analysis, it is calculated that the proposed MR-KM-DCNN is greater than the existing SSL-DCNN, AN-DCNN and KM-DCNN. So, it is proved that the proposed method can achieve better ROC compared to all other existing methods for predicting BC images.

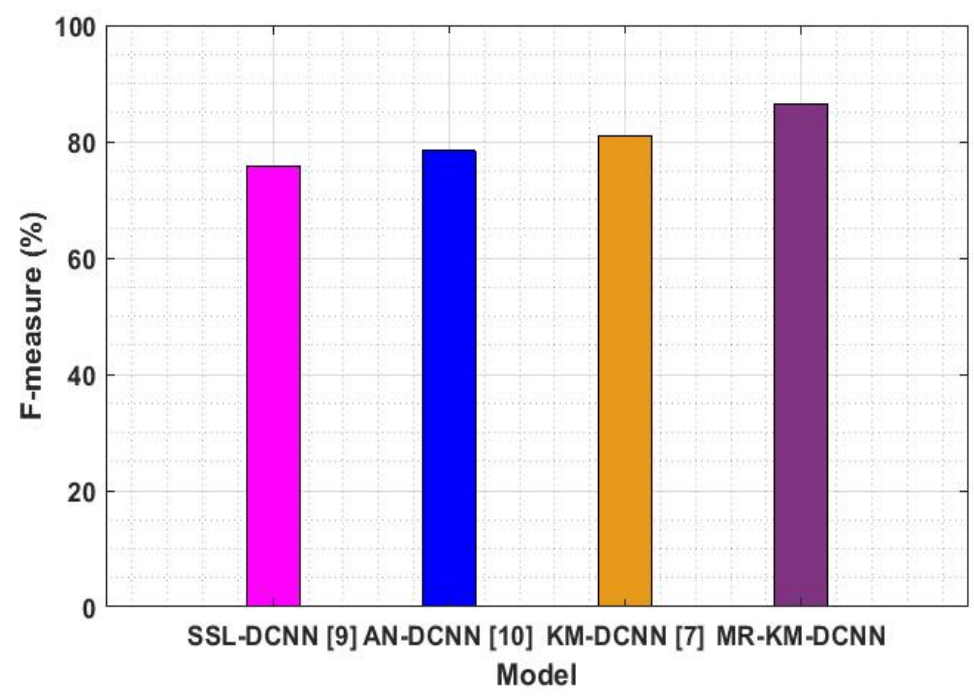

Figure. 5 Comparison of F-measure values 


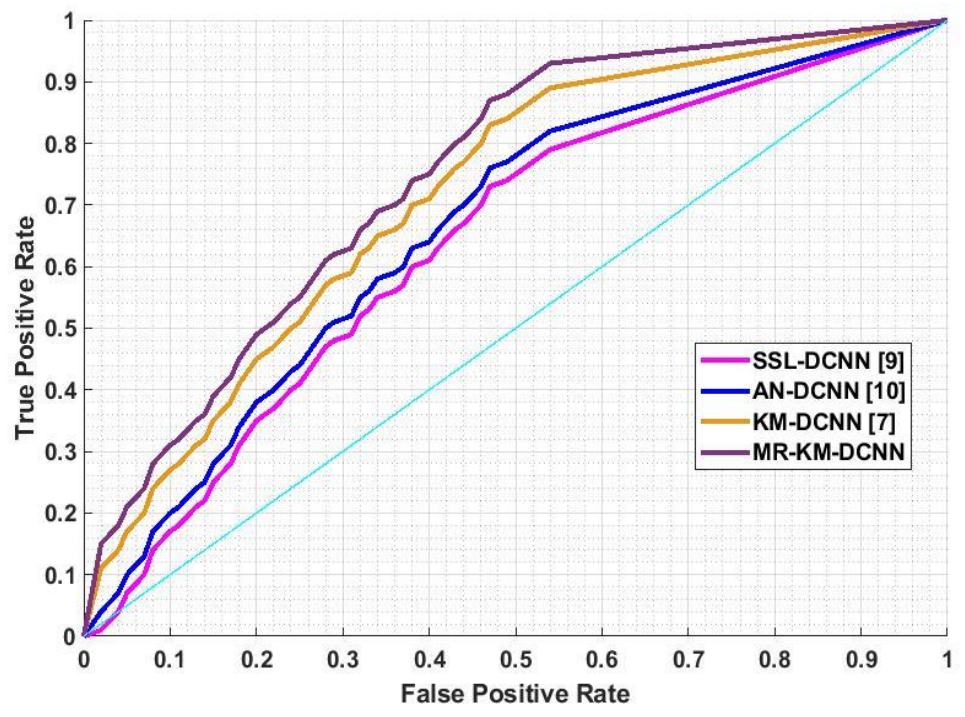

Figure. 6 Comparisons of ROC values

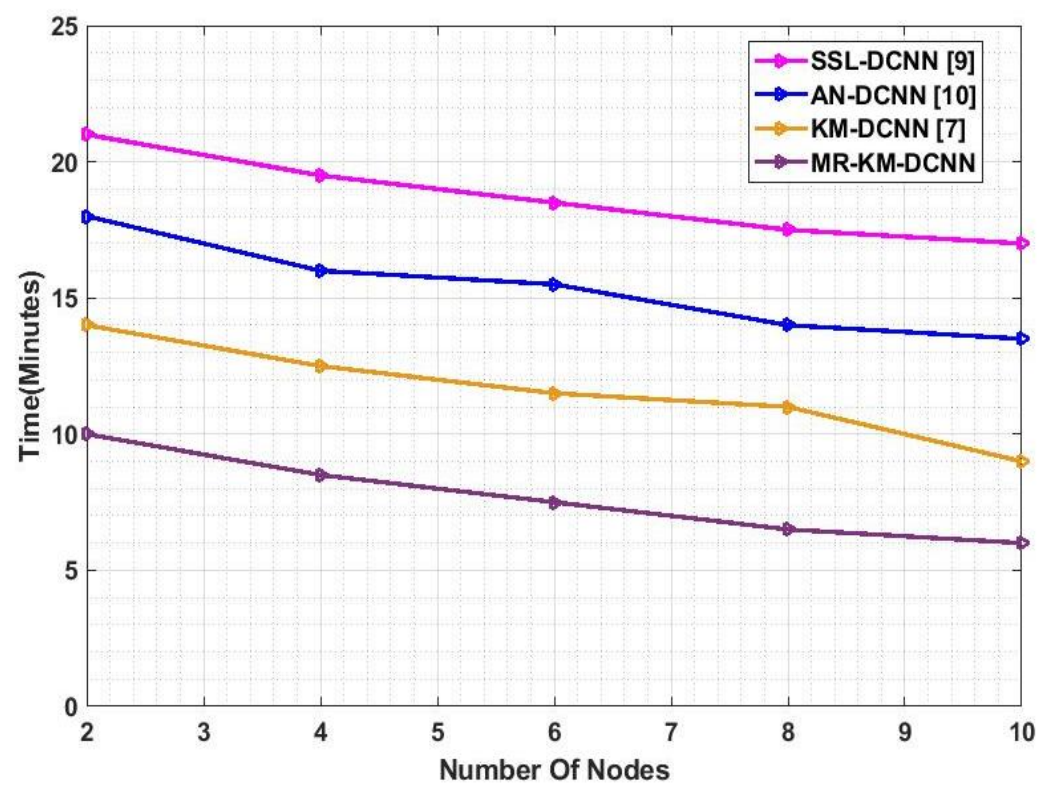

Figure. 7 Comparison of processing time

Fig. 7 illustrates the comparison of processing time among SSL-DCNN, AN-DCNN, KM-DCNN and MR-KM-DCNN methods. The other methods run on different nodes without using the MR framework. Instead, the data is distributed manually and run on various numbers of nodes. The averaged time taken from independent nodes is represented in the graph. MR-KM-DCNN is run in the MR framework with various numbers of nodes. From this analysis, it is found that the processing time of proposed MR-KMDCNN is lesser than other existing method.

\section{Conclusion}

DL algorithms have been affected by the growth of large amounts of information and increasing task difficulties, leading to long learning epochs and significant computing costs. In order to address more complicated scenarios, learned classifiers should retain high efficiency and scalability while remaining cost-effective. To that purpose, this 
classification accuracy while also being close to linear in terms of speedup. Finally, the experimental results prove that the proposed MR-KM-DCNN method achieves an accuracy of $88.35 \%$. Similarly, the precision, recall and f-measure values of proposed method are $85.27 \%, 87.86 \%$ and $86.55 \%$ which is higher than existing methods. The running time of the method is reduced $60 \%$ average. Thus, the findings exhibit that the MR structure is a powerful system for improving DCNN performance and scalability. The proposed MR-KM-DCNN method effectively detects BC detection in Mammogram images. The further image processing part of breast images can be incorporated into the MR framework in the future.

\section{Conflicts of interest}

The authors declare no conflict of interest.

\section{Author contributions}

The paper conceptualization, methodology, software, validation, formal analysis, investigation, resources, data curation, writing - original draft preparation, writing-review and editing, visualization, have been done by $1^{\text {st }}$ author. The supervision and project administration, have been done by $2^{\text {nd }}$ and $3^{\text {rd }}$ author.

\section{References}

[1] M. R. Ataollahi, J. Sharifi, M. Paknahad, and A. Paknahad, "Breast cancer and associated factors: a review", Journal of medicine and life, Vol.8, No. 4, pp. 6-11, 2015.

[2] G. N. Sharma, R. Dave, J. Sanadya, P. Sharma, and K. K. Sharma, "Various types and management of breast cancer: an overview", $J$ Adv Pharm Technol Res, Vol.1, No.2, pp.10926, 2010.

[3] P. A. Baltzer, P. Kapetas, M. A. Marino, and P. Clauser, "New diagnostic tools for breast cancer", Memo-Magazine of European Medical Oncology, Vol. 10, No. 3, pp. 175-180, 2017.

[4] F. Sadoughi, Z. Kazemy, F. Hamedan, L. Owji, M. Rahmanikatigari, and T. T. Azadboni, "Artificial intelligence methods for the diagnosis of breast cancer by image processing: a review", Breast Cancer: (Dove Medical Press), Vol. 10, pp. 219-230, 2018.
[5] F. Hong, F. Hongyu, L. Shan, Q. Zhang, and R. S. Fatima, "Automatic breast cancer detection based on optimized neural network using whale optimization algorithm", International Journal of Imaging Systems and Technology, Vol. 31, No. 1, pp. 425-438, 2020.

[6] A. Rakhlin, A. Shvets, V. Iglovikov, and A. A. Kalinin "Deep Convolutional neural networks for breast cancer histology image analysis", Image analysis and recognition. ICIAR 2018. Lecture notes in computer science, Vol. 10882, pp. 737-744, 2018.

[7] P. Kaur, G. Singh, and P. Kaur, "Intellectual detection and validation of automated mammogram breast cancer images by multiclass SVM using deep learning classification", Informatics in Medicine Unlocked, Elsevier journal", Vol. 16, pp. 100151, 2019.

[8] Q. Zhang, Y. Xiao, W. Dai, J. Suo, C. Wang, J. Shi, and H. Zheng, "Deep learning based classification of breast tumors with shear-wave elastography", Ultrasonics, Vol. 72, pp. 150157, 2016

[9] W. Sun, T. L. B. Tseng, J. Zhang, and W. Qian "Enhancing deep Convolutional neural network scheme for breast cancer diagnosis with unlabeled data", Computerized Medical Imaging and Graphics, Vol. 57, pp. 4-9. 2017.

[10] S. A. Hassan, M. S. Sayed, M. I. Abdalla, and M. A. Rashwan,"Breast cancer masses classification using deep convolutional neural networks and transfer learning", Multimed Tools Appl , Vol. 79, pp. 30735-30768,2020.

[11] G. Wadhwa and A. Kaur, "A Deep CNN Technique for Detection of Breast Cancer Using Histopathology Images", IEEE Advanced Computing and Communication Technologies for High Performance Applications (ACCTHPA), pp. 179-185, 2020.

[12] M A. A. Masni, M. A. A. Antari, J. M. Park, G. Gi, T. Y. Kim, P. Rivera, and T. S. Kim, "Simultaneous detection and classification of breast masses in digital mammograms via a deep learning YOLO-based CAD system", Computer methods and programs in biomedicine, Vol.157, pp. 85-94, 2018

[13] M. Saha, C. Chakraborty, and D. Racoceanu, "Efficient deep learning model for mitosis detection using breast histopathology images", Computerized Medical Imaging and Graphics, Vol. 64, pp. 29-40, 2018.

[14] R. Saturi and P. Premchand, "Multi-Objective Feature Selection Method by Using ACO with PSO Algorithm for Breast Cancer Detection", 
International Journal of Intelligent Engineering and Systems, Vol. 14, No. 5, pp. 359-368, 2021.

[15] P. Mathialagan and M. Chidambaranathan, "Analysis and Classification of H\&E-Stained Oral Cavity Tumour Gradings Using Convolution Neural Network", International Journal of Intelligent Engineering and Systems, Vol. 14, No. 5, pp. 517-528, 2021.

[16] W. L. A. Yaseen, A. Jehad, Q. A. Abed, and A. K. Idrees, "The Use of Modified K-Means Algorithm to Enhance the Performance of Support Vector Machine in Classifying Breast Cancer", International Journal of Intelligent Engineering and Systems, Vol. 14, No. 2, pp. 190-200, 2021.

[17] N. R. P. Pullaiah, D. Venkatasekhar, P. Venkatramana, and B. Sudhakar, "Detection of Breast Cancer on Magnetic Resonance Imaging Using Hybrid Feature Extraction and Deep Neural Network Techniques", International Journal of Intelligent Engineering and Systems, Vol. 13, No. 6, pp. 229-240, 2020.

[18] E. A. Radhi and M. Y. Kamil, "Breast Tumor Detection Via Active Contour Technique", International Journal of Intelligent Engineering and Systems, Vol.14, No.4, pp. 561-570, 2021.

[19] J. Leung, "MapReduce based Convolutional neural networks (Doctoral dissertation)", 2018.

[20] N. Dhanachandra, K. Manglem, and Y. J. Chanu, "Image Segmentation Using K-means Clustering Algorithm and Subtractive Clustering Algorithm", Procedia Computer Science, Vol. 54, pp. 764-771, 2015.

[21] S. Karen and A. Zisserman, "Very deep Convolutional networks for large-scale image recognition", International Conference on Learning Representations (ICLR), 2015.

[22] K. He, X. Zhang, S. Ren, and J. Sun,"Delving deep into rectifiers: Surpassing human-level performance on imagenet classification", Proceedings of the IEEE international conference on computer vision, pp. 1026-1034, 2015.

[23] http://peipa.essex.ac.uk/info/mias.htm 